\title{
FSA Consumer segmentation
}

Area of research interest: Behaviour and perception

Study duration: 2020-05-01

Project status: Completed

Authors: Lucy King

Conducted by: ZK Analytics and Madano

Date published: 30 September 2021

DOI: https://doi.org/10.46756/sci.fsa.bmo506

For our audiences, it is important to find out how their attitudes and behaviours relating to food safety differ, in order to understand who is more likely to take food safety risks and in what context. This is essential for effective communications and helps us to shape food safety policy.

The audiences in these documents have been created using attitudinal and behavioural segmentation that categorises people based on their attitudes to food and their reported hygiene and food safety behaviours.

\section{England, Northern Ireland and Wales}

PDF

View FSA Consumer Segmentation Research Report as PDF(Open in a new window) (495.81 $\mathrm{KB})$

\section{England, Northern Ireland and Wales}

PDF

View FSA Allergen Segmentation Research Report as PDF(Open in a new window) (225.56 KB) 